\title{
COHOMOLOGY FOR THE ERGODIC ACTIONS OF COUNTABLE GROUPS ${ }^{1}$
}

\author{
JOEL J. WESTMAN
}

\begin{abstract}
Certain aspects of Mackey's theory of virtual groups were fitted into a cohomology theory for ergodic groupoids in a previous paper by the author. Here we relate the groupoid cohomology for ergodic groupoids that arise (by Mackey's construction) from ergodic actions of countable groups to the usual group cohomology. In case the countable group is a free group, we find that the groupoid cohomology in dimension $>1$ is $=\{0\}$.
\end{abstract}

Given an ergodic action of a countable group, $G$, on a standard Borel space, $X$, with $G$-invariant measure class, $\widetilde{C}$, we form the ergodic groupoid $\mathcal{F}$ as in [4, Example 3.2b] (cf. [2]), the transitive ergodic groupoid $X \times G \times X=\mathcal{G}$, and the natural monomorphism, $h: \mathcal{F} \rightarrow \mathcal{G}$ (inclusion map). The group cohomology referred to below is as developed in [3].

Given an abelian Borel group $A$ (as in [4]), we define $A_{1}=\{$ Borel functions, $f: X \rightarrow A\}$ mod equality a.e., with the abelian group structure defined by pointwise addition of functions. The action of $G$ on $A_{1}$ is given by $\gamma(f)=f \cdot \gamma^{-1}$. With the trivial action of $G$ on $A$, the map $A \rightarrow A_{1} ; \alpha \rightarrow$ constant function $\equiv \alpha$, is a $G$-module homomorphism, and we obtain the short exact sequence of $G$-modules

$$
0 \rightarrow A \rightarrow A_{1} \rightarrow A_{1} / A \rightarrow 0 .
$$

$H^{0}(G ; A) \cong H^{0}\left(G ; A_{1}\right)$ since the action of $G$ is ergodic.

1.0. Theorem. The long exact sequence ( $c f$. $[3$, page 116])

$$
\begin{gathered}
0 \rightarrow H^{0}\left(G ; A_{1} / A\right) \rightarrow \cdots \rightarrow H^{n}(G ; A) \rightarrow H^{n}\left(G ; A_{1}\right) \\
\rightarrow H^{n}\left(G ; A_{1} / A\right) \rightarrow H^{n+1}(G ; A) \rightarrow \cdots
\end{gathered}
$$

is isomorphic to the long exact sequence for $h: \mathcal{F} \rightarrow g$ as given in $[4,4.11]$, with $A$ as the coefficient group. In particular, $H^{n}(\mathcal{F} ; A) \cong H^{n}\left(G ; A_{1}\right)$ and $H^{n+1}(h ; A) \cong H^{n}\left(G, A_{1} / A\right)$, for $n \geqq 0$.

Proof. We will write elements of $\mathcal{F}$ as $(x, \gamma)$ instead of $\left(x, \gamma, \gamma^{-1} x\right)$,

Received by the editors August 7, 1969.

AMS 1969 subject classifications. Primary 2870, 2050, 2095.

Key words and phrases. Ergodic groupoid, virtual group, ergodic action, cohomology, countable group.

${ }^{1}$ This research was supported in part by the National Science Foundation under Grant No. GP-8291. 
for convenience. For $n \geqq 0$ there is a natural Borel isomorphism

$$
\begin{gathered}
\mathcal{F}^{n} \rightarrow X \times G \times \underset{n}{G} \underset{\mathrm{copies}}{\times} \times G=X \times G^{n}, \\
\left(\left(x, \gamma_{0}\right),\left(\gamma_{0}^{-1} x, \gamma_{1}\right), \cdots,\left(\gamma_{n-2}^{-1} \cdots \gamma_{0}^{-1} x, \gamma_{n-1}\right)\right) \rightarrow\left(x, \gamma_{0}, \gamma_{1}, \cdots, \gamma_{n-1}\right) .
\end{gathered}
$$

We replace $\mathfrak{F}^{n}$ by $X \times G^{n}$ by this map, from here on. Let $A_{B}=\{$ Borel functions: $X \rightarrow A\}$. Then the maps, $\phi^{n}$,

$$
\begin{gathered}
\phi^{n}: C_{B}^{n}(\mathcal{F} ; A) \rightarrow C^{n}\left(G ; A_{B}\right) ; \\
\phi^{n}(f)\left(\gamma_{0}, \gamma_{1}, \cdots, \gamma_{n-1}\right)(x)=f\left(x, \gamma_{0}, \gamma_{1}, \cdots, \gamma_{n-1}\right)
\end{gathered}
$$

(just the "law of exponents") for $n>0$, and $\phi^{0}: C^{0}(\mathcal{F} ; A)=A_{B}$, are bijective. With the action of $G$ on $A_{B}$ given by $\gamma(f)=f \circ \gamma^{-1}$, we find by direct computation that the $\phi^{n}$ 's commute with the coboundary homomorphisms, and accordingly induce isomorphisms $\phi^{n}: H_{B}^{n}(\mathfrak{F} ; A)$ $\rightarrow H^{n}\left(G ; A_{B}\right)$ for $n \geqq 0$.

Now let $A_{\text {ic }}=\{$ Borel functions, $f: X \rightarrow A$ such that $f=0$ a.e. on $X\}$. Then $\phi^{n}\left(C_{\mathrm{io}}^{n}(\mathcal{F} ; A)\right) \subseteq C^{n}\left(G ; A_{\text {ic }}\right)$ since if $f=0$ on $\left(\mathfrak{F} \uparrow X^{\prime}\right)^{n}$ for an i.c. $F\left\lceil X^{\prime}\right.$ of $\mathcal{F}$ then $\phi^{n}(f)\left(\gamma_{0}, \gamma_{1}, \cdots, \gamma_{n-1}\right)=0$ on $X^{\prime \prime}$, where $X^{\prime \prime}=X^{\prime} \cap \gamma_{0}\left(X^{\prime}\right) \cap \cdots \cap \gamma_{n-1}\left(X^{\prime}\right)$, and $X^{\prime \prime}$ is a null set. Moreover, if $k \in C^{n}\left(G ; A_{\text {ic }}\right)$ and $X_{\bar{\gamma}}=\{x: k(\bar{\gamma})(x)=0\}\left(\bar{\gamma}=\left(\gamma_{0}, \gamma_{1}, \cdots, \gamma_{n-1}\right) \in G^{n}\right)$, let $X^{\prime}=\bigcap_{\bar{\gamma} \in G^{n}} X_{\bar{\gamma}}$. Then $X-X^{\prime}$ is a null set by the countability of $G$, and $\left(\phi^{n}\right)^{-1}(k)=0$ on the i.c. $\mathcal{F} \uparrow X^{\prime}$ of $\mathcal{F}$. Hence $\phi^{n}: C_{\mathrm{ic}}^{n}(\mathcal{F} ; A)$ $\rightarrow C^{n}\left(G ; A_{\text {ic }}\right)$ is bijective.

We obtain the diagram

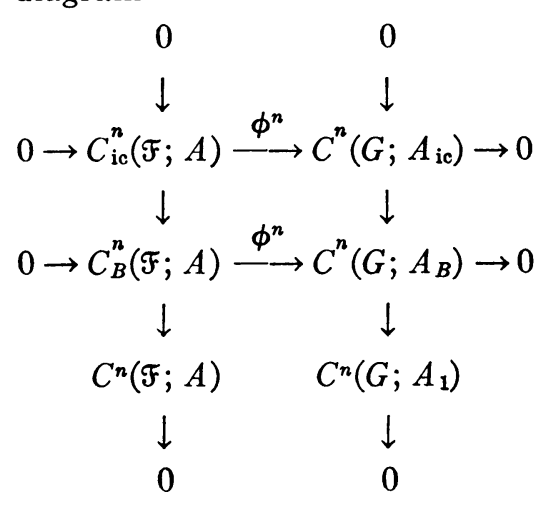

with exact columns and exact top two rows. Then we extend $\phi^{n}$ to an isomorphism $\phi^{n}: C^{n}(F ; A) \rightarrow C^{n}\left(G ; A_{1}\right)$, commuting with the coboundary maps. Accordingly, we obtain the desired isomorphism $\phi^{n}: H^{n}(\mathcal{F} ; A) \rightarrow H^{n}\left(G ; A_{1}\right)$ for $n \geqq 0$.

We note that $H^{1}(h ; A) \cong\left\{\right.$ Borel functions $X \rightarrow A$ such that $f \cdot \gamma^{-1}-f$ $=$ a constant a.e. for each $\gamma \in G\}$ mod constants a.e. $\cong G$ invariant 
elements in $A_{1} / A \cong H^{0}\left(G ; A_{1} / A\right)$. Also, the diagram

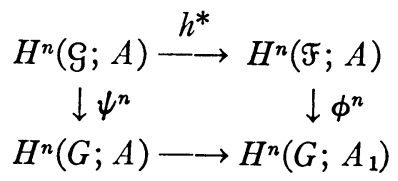

commutes (where $\psi^{n}$ is the isomorphism obtained in [4, Theorem $3.54])$. We then fill in the isomorphisms $H^{n+1}(h ; A) \rightarrow H^{n}\left(G ; A_{1} / A\right)$ for $n>0$ by the 5-lemma to obtain an isomorphism between the relative cohomology sequence for $h: \mathcal{F} \rightarrow \mathcal{G}$ and the group cohomology sequence

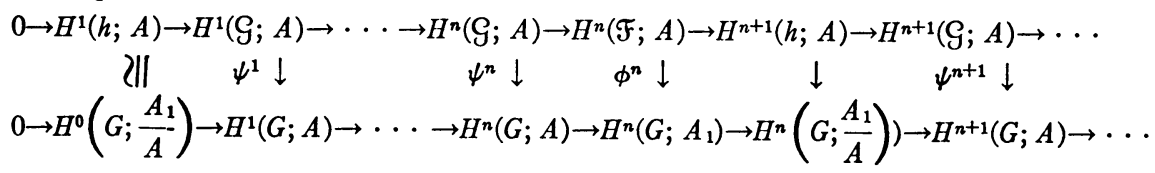

1.1. Corollary. If $G$ is a free countable group, then $H^{i}(\mathfrak{F} ; A)=0$ for $i>1$ and $H^{i}(h ; A)=0$ for $i>2$. In particular, the long exact sequence for $h$ becomes $0 \rightarrow H^{1}(h ; A) \rightarrow H^{1}(G ; A) \rightarrow H^{1}(\mathcal{F} ; A) \rightarrow H^{2}(h ; A) \rightarrow 0$.

Proof. See reference [3, Chapter IV Theorem 7.3, p. 123]. If $G$ is a free group and $B$ is any abelian group, any action of $G$ on $B$, then $H^{n}(G ; B)=0$ for $n>1$.

ExAmple. Let $G$ be a countable group, $H$ a subgroup of $G, X=G / H$, and $A$ an abelian group. Then by Theorem $1, H^{n}\left(G ; A_{1}\right) \cong H^{n}(\mathscr{F} ; A)$. The map $X \times H \times X \rightarrow \mathcal{F} ;(q, \Phi, p) \rightarrow\left(q, \zeta(q) \cdot \Phi \cdot \zeta(p)^{-1}, p\right)$, where $\zeta: X \rightarrow G$ is a section of $\pi: G \rightarrow X ; g \rightarrow g H$, is a groupoid isomorphism. By Theorem 3.54 in [4], $H^{n}(\mathcal{F} ; A) \cong H^{n}(H ; A)$, and hence $H^{n}\left(G ; A_{1}\right)$ $\cong H^{n}(H ; A)$. This result holds for arbitrary groups, $G$, and arbitrary subgroups, $H$, as can be seen by working through the proofs, disregarding the Borel conditions in the theorems and definitions and replacing the "ergodic" requirement by "transitive." Then the result $H^{n}\left(G ; A_{1}\right) \cong H^{n}(H ; A)$ is the special case of Theorem 4 , Chapter $2, \S 3$ in [1] where the action of $G$ on $A$ is trivial.

\section{REFERENCES}

1. S. Lang, Rapport sur la cohomologie des groupes, Benjamin, New York, 1967. MR 35 \#2948.

2. G. W. Mackey, Ergodic theory and virtual groups, Math. Ann. 166 (1966), 187207. MR 34 \#1444.

3. S. Mac Lane, Homology, Die Grundlehren der math. Wissenschaften, Band 114, Academic Press, New York; Springer-Verlag, Berlin, 1963. MR 28 \#122.

4. J. J. Westman, Cohomology for ergodic groupoids, Trans. Amer. Math. Soc. 146 (1969), 465-471. MR 41 \#31.

University of California, Irvine, California 92664 